\section{Identification of time point to best define 'sub-optimal response' following intravitreal ranibizumab therapy for diabetic macular edema based on real- life data}

\begin{abstract}
Purpose To determine the average time-point at which it is best to define 'sub-optimal response' after ranibizumab treatment for diabetic macular edema (DME) based on the data obtained from real-life clinical practice.

Methods In this retrospective observational study, 322 consecutive treatment naïve eyes with DME were treated with three loading doses of intravitreal ranibizumab followed by re-treatment based on decision of the treating physician on a case-by-case basis. The demographic data, clinic-based visual acuity measurements and central subfield thickness (CST) assessed on spectral domain optical coherence tomography (OCT) were evaluated at baseline (month 0), 1, 2, 3, 6, and 12 months. Results On an average, the improvement in visual acuity and CST was first seen after the loading dose. However, the maximal response in terms of proportion of patients with improvement in visual acuity and/ or CST in this cohort was observed at 12 months. Patients who presented with low visual acuity at baseline ( $<37$ ETDRS letters) were unlikely to attain driving vision with ranibizumab therapy.

Conclusions On an average, a 'sub-optimal response' after ranibizumab therapy is best defined at month 12 as patients may continue to improve with treatment.

Eye (2017) 31, 1594-1599; doi:10.1038/eye.2017.111; published online 16 June 2017
\end{abstract}

Received: 9 January 2017 Accepted in revised form: 19 April 2017 Published online: 16 June 2017
Diabetic macular edema (DME) is the most common cause of moderate visual impairment in
I Chatziralli, M Santarelli, N Patrao, L Nicholson, M Zola, R Rajendram, P Hykin and S Sivaprasad patients with diabetic retinopathy and its prevalence varies from 0 to $3 \%$ in patients with recent diagnosis of diabetes increasing to $28 \%$ in patients with diabetes for more than 20 years. ${ }^{1-3}$ Prospective, randomized clinical trials have shown that intravitreal injections of antivascular endothelial growth factor (anti-VEGF) agents are effective in reducing the macular thickness and improving the visual acuity in patients with DME. ${ }^{4-11}$ However, despite strict clinical trial protocol driven treatment criteria, $\sim 50 \%$ of patients treated with anti-VEGF agents for DME have 'persistent' or 'recurrent' edema on optical coherence tomography (OCT) at 12 months despite an improvement in visual acuity in the majority of cases. ${ }^{12-14}$ Together, they represent 'sub-optimal response' to treatment.

Several definitions of 'persistent' or 'recurrent' DME exist. 'Persistent' DME may imply (i) a reduction of $<10 \%$ of central subfield thickness (CST) from baseline or (ii) persistent excess CST above the normative data or above $300 \mu \mathrm{m}$ on spectral domain OCT in clinical practice at 12 months. ${ }^{14,15}$ Similarly, 'recurrent' DME is defined as (i) an increase by at least $10 \%$ from the achieved lowest CST or (ii) an increase above the normative values after achieving normative data at some point during the first 12 months. ${ }^{4,14}$ The prevalence of 'persistent' or 'recurrent' DME (together termed 'sub-optimal response') as per the above definitions after 12-month anti-VEGF therapy in routine clinical practice, where retreatment is not well-defined as in clinical trials, remains unclear. ${ }^{16}$

In addition, these definitions do not often take into account the visual acuity status. In fact, 
change in visual acuity only modestly correlates with change in CST. ${ }^{17}$ For patients, an improvement in visual acuity is surely more important than any structural alteration. Therefore, when we contemplate a switch or addition of therapy, our primary aim should be to ensure that the change in treatment could maximize the gain in visual acuity. As a result, it is important to define 'suboptimal' response in terms of both visual acuity and macular thickness changes. Although sub-analysis of the Protocol I data, incorporating visual acuity and CST has been reported, ${ }^{12,18}$ a similar analysis in a clinical setting is useful, providing new information about the number of patients showing 'sub-optimal' response in terms of both visual acuity and CST, because evaluating treatment decisions in daily practice is less stringent than in clinical trials.

In light of the above, the purpose of this study was to determine the categories of responders following intravitreal ranibizumab treatment for DME in real-life clinical practice to provide the average expected response in different categories of presenting VA and CST.

\section{Materials and methods}

In this retrospective study, records of consecutive treatment naive patients with DME, who received $0.5 \mathrm{mg}$ intravitreal ranibizumab injections between January 2013 and December 2013 at Moorfields Eye Hospital Medical Retina Service, were reviewed. Inclusion criteria were: (i) presence of center-involving DME in the study eye at baseline; (ii) clinic-based visual acuity between 6/60 and 6/6; (iii) CST $>350 \mu \mathrm{m}$ on OCT on Topcon OCT (equivalent to $400 \mu \mathrm{m}$ on Spectralis OCT) and (iv) followup of at least 1 year after the first ranibizumab injection. Patients with history of vitrectomy, prior treatment with laser, uncontrolled glaucoma, uveitis and those lost to follow-up, were excluded from the study. The study adhered to the tenets of the Declaration of Helsinki and was approved by the Institutional Review Board (ROAD reference number 14/047). Written informed consent was obtained from all patients.

We recorded demographic data, the clinic-based visual acuity in Early Treatment Diabetic Retinopathy Study (ETDRS) letters, the CST on OCT (Topcon 3D-OCT 2000) and the injection record at every visit. The visits considered for this study included baseline (month 0$), 1$, 2, 3, 6, and 12 months. All patients underwent comprehensive eye examination, including visual acuity measurement, color fundus images, OCT and fundus fluorescein angiography (FFA) at baseline as per institutional DME protocol, thereafter only visual acuity measurement and OCT were performed, while FFA was repeated at the discretion of each physician.
The treatment protocol included three monthly intravitreal $0.5 \mathrm{mg}$ ranibizumab injections as a loading phase and then PRN treatment, based on decision of the treating physician on a case-by-case basis. Re-treatment criteria included presence of macular fluid on SD-OCT and/or visual acuity change compared to the previous visit. If the decision was not to proceed with further treatment at a clinic visit, such patients were followed up at 4-8 weekly intervals and retreatment decisions were made at these visits. Focal laser and modified grid laser photocoagulation were performed as per standard ETDRS protocol at physician's discretion anytime after the first three ranibizumab injections. Patients requiring panretinal photocoagulation were also treated promptly during this period.

\section{Statistical analysis}

For the description of patients' characteristics at baseline, mean \pm SD was used for continuous variables and counts with percentages for categorical variables. For the longitudinal comparisons of VA and CST between baseline and each time point, the Wilcoxon matched-pairs signed-ranks test was used; given that four comparisons were done, the level of statistical significance was set at $0.05 / 4=0.0125$, according to the Bonferroni correction.

Statistical analysis was performed using SPSS 22.0 (SPSS Inc, Chicago, IL, USA). A $P$ value $<0.05$ was considered as statistically significant, apart from cases where the Bonferroni correction was adopted, as declared above.

\section{Results}

A total of 332 consecutive treatment naive eyes of 278 patients, who were initiated on ranibizumab treatment for DME and followed up for at least 12 months were evaluated for this study. The mean age of patients was $64.5 \pm 11.3$ years. $61.2 \%$ of patients $(n=170)$ were male and $38.8 \%(n=108)$ female. Given that 54 patients $(19.4 \%)$ had bilateral involvement, our analysis was eye-based. The number of eyes with available data regarding visual acuity and OCT at baseline was $332(100 \%)$; at month 1, $323(97.3 \%)$; at month 2, $324(97.6 \%)$; at month 3, 314 $(94.6 \%)$; at month 6, 318 (95.8\%) and at month 12, 312 $(93.8 \%)$. The mean number of injections given at month 12 was $6.7 \pm 2.2$. Ten $(3 \%)$ patients had supplemental macular laser.

\section{Response based on visual acuity}

The mean visual acuity at baseline was $56.4 \pm 15.3$ ETDRS letters and significantly improved to $64.4 \pm 15.0$ ETDRS letters at month 12. Table 1 shows the proportion of 
Table 1 Proportion of eyes with different visual acuity (ETDRS letters) score at baseline and month 1, 2, 3, 6 and 12

\begin{tabular}{|c|c|c|c|c|c|c|}
\hline Visual acuity (ETDRS letters) & 0 (baseline) & Month 1 & Month 2 & Month 3 & Month 6 & Month 12 \\
\hline$>73$ & $43(13 \%)$ & $57(17.7 \%)$ & $74(22.8 \%)$ & $79(25.2 \%)$ & $91(28.6 \%)$ & $108(34.6 \%)$ \\
\hline $54-73$ & $165(49.7 \%)$ & $179(55.4 \%)$ & $178(54.9 \%)$ & $159(50.6 \%)$ & $160(50.3 \%)$ & $144(46.2 \%)$ \\
\hline $37-53$ & $88(26.5 \%)$ & $59(18.3 \%)$ & $50(15.4 \%)$ & $56(17.8 \%)$ & $45(14.2 \%)$ & $32(10.3 \%)$ \\
\hline$<37$ & $36(10.8 \%)$ & $28(8.7 \%)$ & $22(6.8 \%)$ & $20(6.4 \%)$ & $22(6.9 \%)$ & $28(9 \%)$ \\
\hline
\end{tabular}

Table 2 Proportion of eyes in various categories of visual acuity gain over time

\begin{tabular}{lrrrr}
\hline Change in visual acuity from baseline & Month 1 & Month 2 & Month 3 & Month 6 \\
\hline$\geq 10$ letters gain & $50(15.5 \%)$ & $74(22.8 \%)$ & $94(29.9 \%)$ & $101(30.8 \%)$ \\
5-9 letters gain & $82(25.4 \%)$ & $110(34.0 \%)$ & $80(25.5 \%)$ & $82(25.0 \%)$ \\
$<5$ letters gain & $96(29.7 \%)$ & $79(24.4 \%)$ & $67(21.3 \%)$ & $68(21.4 \%)$ \\
\hline
\end{tabular}

patients classified to various categories of visual acuity at different time-points in the whole cohort. A third of patients achieved driving vision in the treated eye (defined as better than 73 ETDRS letters) at 12 months and increased significantly compared to baseline (13 vs $34.6 \%$ for baseline and month 12 , respectively, $P<0.01$ ). The proportion of patients achieving this outcome gradually increased at every visit. In addition, the proportion of eyes that met the criteria of moderate visual impairment (37-53 ETDRS letters) continued to decline at each visit from $26.5 \%$ at baseline to $10.3 \%$ at 12 months. Therefore, if visual acuity is utilized as the parameter to determine response to therapy, month 12 would be the best time-point to evaluate the effect of the therapy.

Table 2 shows the proportion of patients with change in visual acuity classified into various categories at different time points. The earliest response in terms of visual acuity gains is noted after the loading phase of the three monthly injections. However, in all categories of improvement in visual acuity, the proportion of patients with stable visual acuity (0-4 letters change) continued to decrease with a reciprocal increase in patients with improvement in visual acuity ( $\geq 5$ letters) by month 12 . A total of 174 eyes (55.4\%) and 189 eyes $(60.6 \%)$ gained $>5$ letters at month 3 and month 12, respectively, while 44 eyes (14.0\%) and 62 eyes (19.9\%) gained <15 letters at month 3 and month 12, respectively.

In order to assess the individual response to ranibizumab over time, we also categorized the response rates based on visual acuity gain and evaluated the course of response at month 6 and month 12, using the definitions of the sub-analysis of Protocol I data used in the EARLY study. ${ }^{18}$ About $21 \%$ of eyes showed limited early response with gain in visual acuity of 0-4 ETDRS letters at month 3 , while $10 \%$ of eyes in this category gained more than 10 letters at month 6 and 12. In addition, about $25 \%$ of patients had an intermediate early

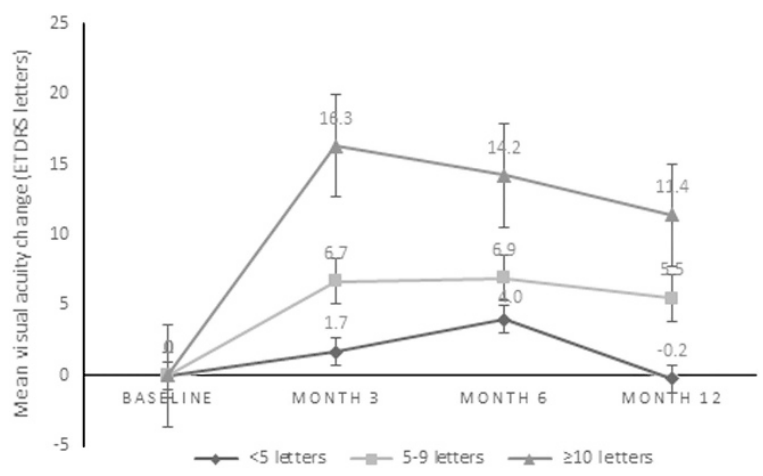

Figure 1 Graph showing the evolution of mean visual acuity change over time in patients with different response at month 3.

response of 5-9 ETDRS letters gain at month 3 and about $30 \%$ of patients showed strong early response after the 3 loading injections with $\geq 10$ ETDRS letters gain, as it is shown on Table 2.

Figure 1 shows the mean change in visual acuity in the three groups of visual acuity gain. Patients, who showed early and strong response at month 3 , were found to gain about 11.4 ETDRS letters at month 12, while patients with 'sub-optimal' response at month 3 presented about 0.2 ETDRS letters loss in mean visual acuity at month 12. However, it is worthy to note that in the category of 'suboptimal' response (<5 ETDRS letters gain), about $25 \%$ of patients had visual acuity gain of more than 10 letters with current treatment at month 12 , as it is shown on Table 3.

At an individual value, the ceiling effect was observed in patients who present with a visual acuity of more than 73 letters. Approximately $14 \%$ of patients in this category dropped to the less level of visual acuity (56-73 letters). In all other categories of visual acuity, most patients remained in the same category or improved. 
Table 3 Proportion of patients with 'sub-optimal' response at month 3 and their evolution over time

\begin{tabular}{llr}
\hline 3 months & 6 months $\mathrm{n}(\%)$ & 12 months $\mathrm{n}(\%)$ \\
\hline$<5$ letters gain $(21.3 \%$ of the study sample) & $<5$ letters gain $28 / 67(41.8 \%)$ & $<5$ letters gain $18 / 67(26.9 \%)$ \\
& $5-9$ letters gain $19 / 67(28.4 \%)$ & $5-9$ letters gain $18 / 67(26.9 \%)$ \\
& $\geq 10$ letters gain $11 / 67(16.4 \%)$ & $\geq 10$ letters gain $16 / 67(23.9 \%)$ \\
\hline
\end{tabular}

Table 4 Proportion of eyes with different central subfield thickness (CST) at baseline and month 1, 2, 3, 6 and 12

\begin{tabular}{|c|c|c|c|c|c|c|}
\hline $\operatorname{CST}(\mu m)$ & Baseline & Month 1 & Month 2 & Month 3 & Month 6 & Month 12 \\
\hline$\leq 300 \mu \mathrm{m}$ & $0(0 \%)$ & $86(26.6 \%)$ & $110(34 \%)$ & $134(42.7 \%)$ & $138(43.4 \%)$ & $146(46.8 \%)$ \\
\hline $301-400 \mu \mathrm{m}$ & $107(32.3 \%)$ & $125(38.7 \%)$ & $133(41.1 \%)$ & $108(34.4 \%)$ & $102(32.1 \%)$ & $92(29.5 \%)$ \\
\hline $401-500 \mu \mathrm{m}$ & $120(36.1 \%)$ & $77(23.8 \%)$ & $53(16.4 \%)$ & $46(14.6 \%)$ & $47(14.8 \%)$ & $42(13.5 \%)$ \\
\hline$\geq 501 \mu \mathrm{m}$ & $105(31.6 \%)$ & $35(10.8 \%)$ & $28(8.6 \%)$ & $26(8.3 \%)$ & $31(9.7 \%)$ & $32(10.3 \%)$ \\
\hline Lowest CST value achieved & $9(2.7 \%)$ & $16(5.0 \%)$ & $42(13.0 \%)$ & $77(24.5 \%)$ & $81(25.5 \%)$ & $107(34.3 \%)$ \\
\hline At least $10 \%$ decrease in CST from baseline & & $205(65.3 \%)$ & $247(76.2) \%$ & $244(78.7 \%)$ & $239(76.4 \%)$ & $241(78 \%)$ \\
\hline
\end{tabular}

None of the patients who present with visual acuity of less than 37 letters achieved more than 73 letters.

\section{Response based on central subfield thickness}

The mean CST was $468.4 \pm 113.3 \mu \mathrm{m}$ at baseline compared to $336.1 \pm 123.4 \mu \mathrm{m}$ at month $12(P<0.001)$. Table 4 shows the proportion of eyes in different CST categories over time. The prevalence of persistent DME (CST $>300 \mu \mathrm{m})$ at month 12 was $53.4 \%$. The proportion of eyes with CST $\leq 300 \mu \mathrm{m}$ increased at every visit during the loading phase of 3 injections and then remained stable from month 3 to month 12. Moreover, the proportion of eyes with CST between 300 and $400 \mu \mathrm{m}$ remained stable throughout the 12 months. On the contrary, the proportion of eyes with CST more than $400 \mu \mathrm{m}$ decreased to a third by 12 months. It is worthy to note that $34.4 \%$ of eyes (the highest proportion) achieved the lowest CST value at month 12 . When we consider the $26(8.3 \%)$ patients with $>500 \mu \mathrm{m}$ at month 3, 3 patients $(11.5 \%)$ presented CST $<300 \mu \mathrm{m}$ at month 6 and 4 patients (15.4\%) at month 12 .

It is worthy to note that in patients with visual acuity $>73$ ETDRS letters, $51 \%$ of eyes presented CST $>300 \mu \mathrm{m}$ at month 12 , showing that persistent DME may exist despite the 'good' visual acuity. In addition, if one takes into account both visual acuity and CST, in this study, only 0.7 and $4.4 \%$ of eyes achieved a visual acuity of $6 / 6$ and CST $\leq 300 \mu \mathrm{m}$ at month 3 and month 12 respectively, suggesting that in real-life attainment visual acuity of $6 / 6$ with resolution of fluid is a challenging task to achieve.

\section{Discussion}

The principal message of this study is that based on the definitions used to determine response to ranibizumab treatment for DME, the average time-point to consider 'sub-optimal' response to ranibizumab therapy is at month 12 as most patients continue to improve visual acuity over the 12 months' period. However, individual discretions may apply. For example, our data indicate that identifying 'sub-optimal' response after the loading phase may be more appropriate in patients with low visual acuity at baseline ( $<37$ ETDRS letters).

Currently, there are no robust data to suggest that switch from one anti-VEGF therapy to another results in visual acuity gain and/or better resolution of macular fluid. On the basis of our experience in neovascular age related macular degeneration (AMD), a switch from ranibizumab to aflibercept resulted in anatomical improvement with resolution of macular fluid with no visual acuity gain. ${ }^{19}$ However, given that the two diseases are different in pathophysiology, there are no exact data on switching between anti-VEGF agents in DME. In addition, Ferris et $a l^{20}$ reported that due to the 'regression to the mean' phenomenon, it is difficult to assess the impact of switch from one to another anti-VEGF without a control group, and found that patients with DME and AMD presented increase in visual acuity even if they continued the same treatment and did not switch therapy by analyzing a subgroup of CATT and DRCR.net study. This study provides evidence of the same in real-life, in patients continuing on ranibizumab for 12 months despite $<5$ letters gain at month 3 , since $\sim 30 \%$ of them gained $\geq 5$ letters subsequently in this cohort. Therefore, in a clinical trial, as switch to another agent should provide a superior response to this to prove the beneficial effect of the second agent.

The study also shows that the increase in visual acuity in patients with DME is a gradual process and the peak in visual acuity may be established only after 6-9 months or longer following initiation of treatment. ${ }^{21}$ Very few 
patients show 'sub-optimal' response after three loading injections and therefore, in general, it is better to wait longer to identify the true 'sub-optimal' responders. There may be exceptions to the rule that one could decide on a case-by-case basis.

Furthermore, there are few clinical trials on the role of switching from anti-VEGF to steroid therapy or addition of steroid therapy to on-going anti-VEGF therapy in DME. ${ }^{22}$ Both the OZLASE and OZDRY studies included patients with long-standing and persistent edema despite previous laser and/or anti-VEGF therapy. ${ }^{23,24}$ These studies did not show any visual benefit despite resolution of fluid at the end of the follow-up, using intravitreal dexamethasone implant (Ozurdex, Irvine, CA, USA). However, there are other studies, showing that intravitreal dexamethasone implant and intravitreal fluocinolone acetonide (lluvien, Alpharetta, GA, USA) are effective in improving visual acuity and reducing macular thickness in patients with DME refractory to previous anti-VEGF therapy. ${ }^{25-27}$ Randomized controlled trials are needed to confirm or disprove that a switch to another therapy can improve visual acuity in patients who are defined as 'sub-optimal responders' to anti-VEGF therapy.

Another interesting finding of our study was that unlike clinical trials, in real-life clinical practice, treatment decisions seemed to be more conservative. In clinical practice, the less stringent re-treatment criteria may be attributed to multiple factors, including tendency of clinicians to under-treat, patients' reluctance to have continuous regimen of repeated injections or due to strain on services to provide prompt and timely treatment. Therefore, a change in the initial treatment or use of combined therapy is often contemplated despite lack of evidence.

Potential limitation of this study is its retrospective nature. Moreover, it should be mentioned that visual acuity assessment was clinic-based. However, this study has a relatively large sample size and provides real-life data of a tertiary retina center.

In conclusion, in most instances, month 12 is an ideal time-point for identifying 'sub-optimal' treatment response after ranibizumab therapy, although month 3 may be more appropriate in patients with low visual acuity at baseline ( $<37$ ETDRS letters).

\section{Summary}

\section{What was known before}

- There is no general consensus about when to switch ranibizumab treatment for diabetic macular edema and who were 'non-responders' to treatment.

What this study adds

- A patient with 'sub-optimal response' after ranibizumab therapy for diabetic macular edema is best defined at month 12 as patients may continue to improve with treatment.

\section{Conflict of interest}

$\mathrm{PH}$ and SS have received travel grants, research grants and speaker fees and attended advisory board meetings of Bayer, Allergan and Novartis.

\section{References}

1 Klein R, Klein BE, Moss SE. Visual impairment in diabetes. Ophthalmology 1984; 91: 1-9.

2 Zhang X, Saaddine JB, Chou CF, Cotch MF, Cheng YJ, Geiss LS et al. Prevalence of diabetic retinopathy in the United States, 2005-2008. JAMA 2010; 304: 649-656.

3 Klein R, Klein BE, Moss SE, Davis MD, DeMets DL. The Wisconsin epidemiologic study of diabetic retinopathy. IV. Diabetic macular edema. Ophthalmology 1984; 91: 1464-1474.

4 Nguyen QD, Shah SM, Khwaja AA, Channa R, Hatef E, Do DV et al. Two-year outcomes of the ranibizumab for edema of the mAcula in diabetes (READ-2) study. Ophthalmology 2010; 117: 2146-2151.

5 Massin P, Bandello F, Garweg JG, Hansen LL, Harding SP, Larsen $\mathrm{M}$ et al. Safety and efficacy of ranibizumab in diabetic macular edema (RESOLVE Study): a 12-month, randomized, controlled, double-masked, multicenter phase II study. Diabetes Care 2010; 33: 2399-2405.

6 Mitchell P, Bandello F, Schmidt-Erfurth U, Lang GE, Massin P, Schlingemann RO et al. The RESTORE study: ranibizumab monotherapy or combined with laser versus laser monotherapy for diabetic macular edema. Ophthalmology 2011; 118: 615-625.

7 Brown DM, Nguyen QD, Marcus DM, Boyer DS, Patel S, Feiner $\mathrm{L}$ et al. Long-term outcomes of ranibizumab therapy for diabetic macular edema: the 36-month results from two phase III trials: RISE and RIDE. Ophthalmology 2013; 120: 2013-2022.

8 Elman MJ, Ayala A, Bressler NM, Browning D, Flaxel CJ, Glassman AR et al. Intravitreal Ranibizumab for diabetic macular edema with prompt versus deferred laser treatment: 5-year randomized trial results. Ophthalmology 2015; 122: 375-381.

9 Korobelnik JF, Do DV, Schmidt-Erfurth U, Boyer DS, Holz FG, Heier JS et al. Intravitreal aflibercept for diabetic macular edema. Ophthalmology 2014; 121: 2247-2254.

10 Brown DM, Schmidt-Erfurth U, Do DV, Holz FG, Boyer DS, Midena E et al. Intravitreal Aflibercept for Diabetic Macular Edema: 100-Week Results From the VISTA and VIVID Studies. Ophthalmology 2015; 122: 2044-2052.

11 Wells JA, Glassman AR, Ayala AR, Jampol LM, Bressler NM, Bressler SB et al. Aflibercept, bevacizumab, or ranibizumab for diabetic macular edema: two-year results from a comparative effectiveness randomized clinical trial. Ophthalmology 2016; 123: 1351-1359.

12 Bressler SB, Qin H, Beck RW, Chalam KV, Kim JE, Melia M et al. Factors associated with changes in visual acuity and central subfield thickness at 1 year after treatment for diabetic macular edema with ranibizumab. Arch Ophthalmol 2012; 130: 1153-1161.

13 Wells JA, Glassman AR, Ayala AR, Jampol LM, Aiello LP, Antoszyk AN et al. Aflibercept, bevacizumab, or ranibizumab for diabetic macular edema. N Engl J Med 2015; 372: 1193-1203.

14 Bressler SB, Ayala AR, Bressler NM, Melia M, Qin H, Ferris FL 3rd et al. Persistent macular thickening after 
ranibizumab treatment for diabetic macular edema with vision impairment. JAMA Ophthalmol 2016; 134: 278-285.

15 Apte RS. What is chronic or persistent diabetic macular edema and how should it be treated? JAMA Ophthalmol 2016; 134: 285-286.

16 Aiello LP, Beck RW, Bressler NM, Browning DJ, Chalam KV, Davis $\mathrm{M}$ et al. Rationale for the diabetic retinopathy clinical research network treatment protocol for center-involved diabetic macular edema. Ophthalmology 2011; 118: e5-14.

17 Browning DJ, Glassman AR, Aiello LP, Beck RW, Brown DM, Fong DS et al. Relationship between optical coherence tomography-measured central retinal thickness and visual acuity in diabetic macular edema. Ophthalmology 2007; 114: 525-536.

18 Gonzalez VH, Campbell J, Holekamp NM, Kiss S, Loewenstein A, Augustin AJ et al. Early and long-term responses to anti-vascular endothelial growth factor therapy in diabetic macular edema: analysis of protocol I data. Am J Ophthalmol 2016; 172: 72-79.

19 Chatziralli I, Nicholson L, Vrizidou E, Koutsiouki C, Menon D, Sergentanis TN et al. Predictors of outcome in patients with neovascular age-related macular degeneration switched from ranibizumab to 8-weekly aflibercept. Ophthalmology 2016; 123: 1762-1770.

20 Ferris FL 3rd, Maguire MG, Glassman AR, Ying GS, Martin DF. Evaluating effects of switching anti-vascular endothelial growth factor drugs for age-related macular degeneration and diabetic macular edema. JAMA Ophthalmol 2016.

21 Ziemssen F, Schlottman PG, Lim JI, Agostini H, Lang GE, Bandello F. Initiation of intravitreal aflibercept injection treatment in patients with diabetic macular edema: a review of VIVID-DME and VISTA-DME data. Int I Retina Vitreous 2016; $2: 16$.

22 Hussain RM, Ciulla TA. Treatment strategies for refractory diabetic macular edema: switching anti-VEGF treatments, adopting corticosteroid-based treatments, and combination therapy. Expert Opin Biol Ther 2016; 16: 365-374.

23 Heng LZ, Sivaprasad S, Crosby-Nwaobi R, Saihan Z, Karampelas $\mathrm{M}$, Bunce $\mathrm{C}$ et al. A prospective randomised controlled clinical trial comparing a combination of repeated intravitreal Ozurdex and macular laser therapy versus macular laser only in centre-involving diabetic macular oedema (OZLASE study). Br J Ophthalmol 2016; 100: 802-807.

24 Ramu J, Yang Y, Menon G, Bailey C, Narendran N, Bunce C et al. A randomized clinical trial comparing fixed vs pro-renata dosing of Ozurdex in refractory diabetic macular oedema (OZDRY study). Eye 2015; 29: 1603-1612.

25 Khan Z, Kuriakose RK, Khan M, Chin EK, Almeida DR. Efficacy of the intravitreal sustained-release dexamethasone implant for diabetic macular edema refractory to antivascular endothelial growth factor therapy: meta-analysis and clinical implications. Ophthalmic Surg Lasers Imaging Retina 2017; 48: 160-166.

26 Pacella F, Romano MR, Turchetti P, Tarquini G, Carnovale A, Mollicone A et al. An eighteen-month follow-up study on the effects of Intravitreal Dexamethasone Implant in diabetic macular edema refractory to anti-VEGF therapy. Int $\mathrm{J}$ Ophthalmol 2016; 9: 1427-1432.

27 Elaraoud I, Andreatta W, Kidess A, Bhatnagar A, Tsaloumas M, Quhill F et al. Use of flucinolone acetonide for patients with diabetic macular oedema: patient selection criteria and early outcomes in real world setting. BMC Ophthalmol 2016; 16: 3. 